\title{
L'ethique de la communication des organisations : de la dénonciation à la reconnaissance
}

Gilles Gauthier

\section{(2) OpenEdition}

12 Journals

Édition électronique

URL : http://journals.openedition.org/communicationorganisation/1593

DOI : 10.4000/communicationorganisation. 1593

ISSN : $1775-3546$

Éditeur

Presses universitaires de Bordeaux

Édition imprimée

Date de publication : 1 novembre 1992

ISSN : 1168-5549

Référence électronique

Gilles Gauthier, «L'ethique de la communication des organisations : de la dénonciation à la

reconnaissance », Communication et organisation [En ligne], 2 | 1992, mis en ligne le 26 mars 2012,

consulté le 01 mai 2019. URL : http://journals.openedition.org/communicationorganisation/1593 ;

DOI : 10.4000/communicationorganisation.1593

Ce document a été généré automatiquement le 1 mai 2019.

(c) Presses universitaires de Bordeaux 


\title{
L'ethique de la communication des organisations : de la dénonciation à la reconnaissance
}

\author{
Gilles Gauthier
}

... just as a poorly knit sweater can unravel at the first hard tug, an integrated theory of public relations is likely to unravel at the first hard case'if ethics is not a part of its theoretical foundation.

Catherine A. Pratt et Terry Lynn Reutner

1 Les chercheurs et analystes de la communication ont-ils raison de réduire le grand intérêt que manifestent aujourd'hui les entreprises et autres organisations à l'égard de la dimension morale de leurs activités, en particulier de communication, à une stratégie manipulatoire ? Est-il séant, du point de vue «scientifique » qui devrait être le leur, que les «théoriciens" se contentent de critiquer sinon plus carrément de dénoncer la préoccupation éthique des "praticiens » sans auparavant vraiment prendre la peine d'examiner sérieusement la possibilité qu'elle puisse avoir trait à quelque contenu consistant? Est-il justifiable que cette dénonciation serve de prétexte à un rejet plus ou moins déguisé du projet d'une théorie éthique de la communication, et plus spécifiquement de la communication des organisations ${ }^{1}$

2 Le présent texte a pour objectif de contribuer à contrer cet escamotage. Il voudrait donc ouvrir à la reconnaissance de l'éthique de la communication des organisations.

3 Il est très certainement vrai que l'éthique est, dans les faits, parfois utilisée à des fins de manipulation par des entreprises. Il est vrai, également, que tout recours à l'éthique peut cacher une volonté de moraliser les choses à son profit. Il est vrai, enfin, que l'éthique est aujourd'hui à ce point à la mode qu'il en est fourni toutes sortes de définitions extrêmement variées et pour beaucoup injustifiées ${ }^{2}$. Mais ces constats ne devraient pas empêcher de profiter de l'occasion que la popularité de l'éthique offre de faire progresser la discussion sur la conduite des organisations et, aussi, d'esquisser le projet d'une 
éthique de la communication des organisations. Peut-être même l'intensité actuelle de la préoccupation éthique des entreprises et autres organisations est-elle l'expression d'un véritable besoin qu'il conviendrait de pousser à ses limites. Est-ce faire montre de trop de naïveté que de considérer qu'il y a là, possiblement, une chance de modifier pour le mieux le mode de fonctionnement des organisations?

4 La thèse précise dont j'entends faire la démonstration est que la grande et grave difficulté qui se posait jusqu'à maintenant à une pleine reconnaissance de l'éthique de la communication des organisations et qui fonde la dépréciation qu'on en fait, à savoir une opposition entre l'intention morale et la visée organisationnelle, commence à se dissiper.

5 Si l'éthique de la communication des organisations - comme d'ailleurs, plus globalement, l'éthique des organisations tout court - suscite une suspicion telle qu'elle entraîne une réaction critique immédiate, c'est d'abord et avant tout parce qu'elle peut apparaître contradictoire dans les termes : pèse, en effet, sur les organisations un présupposé (ou serait-ce plutôt un préjugé ?) suivant lequel elles poursuivraient une finalité d'efficacité, et même de rentabilité dans le cas des entreprises commerciales et industrielles, indifférente à l'éthique si ce n'est incompatible avec tout sens de la moralité. Autrement dit, organisations et éthique seraient en relation dichotomique de sorte que l'éthique de la communication serait, en elle même, un projet vide de sens dont la recherche ne pourrait donc consister qu'en une manœuvre stratégique.

L'opposition entre organisations et éthique s'amplifie si, comme c'est très souvent le cas, l'on réduit l'éthique au domaine subjectif des préférences morales individuelles. Ainsi que le mettent clairement en évidence des enquêtes menées à ce propos les problèmes éthiques rencontrés par les communicateurs au sein des entreprises et autres types d'organisations sont fréquemment perçus comme générant des pressions pour compromettre leurs valeurs personnelles dans la poursuite d'objectifs corporatifs. Ainsi réduite à l'individualité subjective, l'éthique apparaît encore plus en contradiction avec la nature fonctionnelle des organisations.

7 Or, les choses commencent à changer à cet égard. Je soutiens en tout cas que se font jour un certain nombre d'indices, dans la littérature américaine consacrée au trio organisations - communication - éthique, qui amènent à penser que pourrait bientôt être levée la lourde hypothèque qui mine l'éthique de la communication des organisations. Plus spécifiquement, je propose de considérer que commencent à être remplies cinq conditions qui permettent de mieux lier moralité et organisations et, par là, de justifier l'éthique de la communication des organisations. Ces cinq conditions sont: une reconnaissance de l'inhérence de la dimension morale à la communication, une reconnaissance de la nécessité d'appuyer la recherche éthique sur un fond théorique et même philosophique, une caractérisation de l'analyse du raisonnement éthique, l'affirmation d'une différenciation d'une éthique de la persuasion par rapport à une éthique de l'objectivité et la prise en compte de la notion d'intérêt. Avant de procéder à un examen plus attentif de chacun de ces points, s'imposent quelques considérations générales au sujet de l'éthique de la communication des organisations. 


\section{L'émergence de l'éthique de la communication des organisations}

Il est légitime de considérer que l'éthique de la communication des organisations est à l'heure actuelle, à se constituer en un champ nouveau et original de recherche. Le surgissement de ce secteur résulte en fait de l'union de deux classes plus générales de préoccupations morales : l'éthique de la communication publique et l'éthique des affaires.

9 Les pratiques de communication publique, le journalisme, les relations publiques, la publicité, le discours politique et plus récemment la communication organisationnelle et institutionnelle donnent lieu, depuis un assez grand nombre d'années, à un questionnement intense d'ordre moral ${ }^{3}$. Parallèlement, depuis presqu'aussi longtemps, une préoccupation éthique a vu le jour au sujet de l'ensemble des activités des entreprises et autres organisations à laquelle on peut donner le nom $d$ "éthique des affaires" (business ethics) ${ }^{4}$. Comme, évidemment, la communication - par les relations publiques, la publicité et différents éléments de communication organisationnelle et institutionnelle mais aussi de toutes sortes d'autres façons-constitué un aspect-clé de l'action des organisations, il était écrit que ces deux intérêts éthiques allaient enfanter un domaine spécifique : l'éthique de la communication des organisations.

Un certain nombre d'indicateurs "institutionnels» annoncent cette naissance. Par exemple, quelques importantes revues "savantes" en communication publique et en éthique des affaires ont consacré, tout récemment, un numéro spécial à des questions éthiques : le Journal of Mass Media Ethics (vol. 4, no. 1,1989), le Communication Quaterly (vol. 38, no. 3, 1990), le Journal of Business Communication (vol. 27, no. 3, 1990) et le Bulletin of the Association for Business Communication (vol. 53, no. 3,1990). Dans le même ordre d'idée, deux des ouvrages classiques de l'éthique de la communication publique, le Media Ethics. Cases \& Moral Reasoning de Christians, Rotzoll et Fackler ainsi que le Ethics in Human Communication de Johannesen dans leur dernière édition, parue respectivement en 1991 et 1990, consacrent pour la première fois un développement à l'éthique de la communication des organisations. Murphy et Bloom font de même dans le récent (1990) Social Marketing. Promoting the Causes of Public and Nonprofit Agencies de Fine. Bref, il est clair que les choses bouillonnent autour de ce qu'on peut sans doute déjà commencer à nommer « l'éthique de la communication des organisations ".

11 Dans l'état actuel des choses, ce terme générique désigne en fait l'amalgame des différents travaux poursuivis en éthique des relations publiques, en éthique de la publicité, en éthique de la communication des affaires (business communication ethics), en éthique de la communication organisationnelle et institutionnelle et en éthique du marketing social.

Deux remarques préliminaires peuvent être faites au sujet de l'éclosion de l'éthique de la communication des organisations.

13 D'abord, si le principal chantier de recherche qu'elle établit est l'élaboration d'une théorie morale de l'activité communicationnelle des organisations, elle ouvre également à une seconde perspective heuristique : l'étude du rôle de la communication dans l'éthique des affaires. Certains auteurs, en effet, cherchent moins à traiter des incidences éthiques de la communication des organisations qu'à déterminer comment elle, la communication, pourrait être un instrument moral de ces mêmes organisations. Ils se trouvent alors à 
tenter de fonder l'éthique des affaires dans la communication. Par exemple, Pettit, Vaught et Pulley (1990) considèrent que c'est par l'acquisition d'une compétence communicative, plus spécifiquement d'habilités de communication interpersonnelle (interpersonal communicative skills), que les dirigeants d'entreprises peuvent parvenir à aider leurs employés à solutionner les problèmes éthiques qui se présentent à eux. Un peu dans le même esprit, certains autres auteurs, dont Hunter (1990), affirment que c'est à travers l'éthique de la communication que peut être le mieux abordée l'éthique des affaires. Spencer et Lehman (1990) expliquent cette identification par les exigences d'analyse et d'évaluation intrinsèques à la communication.

Seconde remarque : la prise en compte de la dimension éthique par des chercheurs dont le premier ou initial objet d'examen est le domaine prosaïque des affaires se fait avec un accent tout particulier qu'on peut qualifier de "pragmatique». Ainsi, Lewis et Speck (1990) proposent d'aborder la question éthique de la communication des organisations suivant un point de vue systématique (a more organic approach) et suggèrent de fournir des définitions objectives (objective, clearly definable meaning<s>) et opératoires (working definition<s>). Tout à fait dans le même sens, Pettit, Vaught et Pulley (1990) plaident pour l'établissement de normes morales opérationnelles (workable ethical standard $<$ s>). Il s'agit là, bien sûr, d'un expression d'intention qui reste à être concrétisée. On peut, toutefois, tout de suite annoncer qu'elle est suffisamment consistante pour commander une certaine forme d'examen moral - dont nous allons plus loin plus précisément rendre compte : l'analyse du raisonnement éthique.

\section{La thèse de l'inhérence}

La façon la plus radicale et définitive de justifier le projet d'une éthique de la communication, entre autres choses des organisations, consiste à affirmer la présence intrinsèque d'une variable morale dans toute forme de communication publique. J'appelle cette idée suivant laquelle la communication comporte toujours une dimension éthique la «thèse de l'inhérence ». Il est possible d'en localiser l'expression plus ou moins explicite dans un assez grand nombre d'ouvrages portant sur les pratiques plus traditionnelles de la communication publique ${ }^{5}$.

Il se trouve que c'est également le cas au moins pour quelques textes consacrés spécifiquement à l'éthique de la communication des organisations. Par exemple, Reinsch (1990), effectuant un survol de la recherche éthique en communication des affaires, s'attarde à un certain nombre de travaux où est mise en evidence sa nature ou son caractère intrinsèquement moral: Goodrich (1958), relativement aux techniques rhétoriques; McGuire (1980), au sujet de l'enseignement des styles et formes et Rents et Debs (1987), par rapport à la représentation de la réalité. Reinsch conclut que...

Perhaps some aspects of human knowledge and practice are amoral, but business communication is not among them. Business communication is inherently moral... (267)

17 De la même manière, Jacobi (1990) défend le point de vue que la communication, en particulier celle qui relève de l'écriture technique (professional writing), n'est pas neutre sur le plan éthique du fait qu'elle soit toujours porteuse de valeurs. Ce que ces différents auteurs, chacun à sa manière, cherchent à exprimer, sans que ce soit explicitement dans ces termes, c'est bien la thèse de l'inhérence : l'idée que la question 
éthique est intrinsèque à la communication des organisations. Cette thèse de l'inhérence est d'une portée considérable. Sur le plan épistémologique, elle constitue le fondement ultime de l'entreprise théorique qu'est ou que pourrait être l'éthique de la communication des organisations. Cette dernière constitue ou pourrait constituer un champ légitime de recherche du fait que, pour ainsi dire, toute communication est habitée par la moralité.

Par ailleurs, la thèse de l'inhérence appliquée spécifiquement à la communication des organisations permet de nuancer, partiellement mais tout de même de façon importante, l'antinomie apparente soulignée en début de texte entre éthique et organisations. Au delà d'une première intuition, peut-être convient-il de considérer non pas que la finalité organisationnelle est incompatible avec l'intention morale mais plutôt, plus restrictivement, que la communication des organisations comporte toujours une incidence morale suivant laquelle se présentent des enjeux éthiques qui peuvent être éclaircis dans un sens moral aussi bien qu'immoral.

\section{L'importance des fondements théorico-philosophiques}

L'un des grands points de tension de l'éthique est qu'elle est une entreprise par nature théorique qui, par ailleurs, prétend avoir un champ d'application pratique. L'ambition de l'éthique, c'est de fournir une connaissance objective de la conduite idéale. Elle comporte donc une visée cognitive relative à l'action concrète. Le rapport entre le caractère théorique de l'éthique et sa prétendue portée pratique a souvent, dans le passé, été pensé davantage comme un écart que comme un véritable lien. Aussi n'est-il pas étonnant que l'éthique philosophique ait fait l'objet d'attaques et aussi, plus récemment qu'on ait cherché à instituer ce qu'on appelle une "éthique appliquée». Par là, on veut évidemment désigner un certain type de démarche éthique qui au lieu de consister en des spéculations désincarnées porterait immédiatement sur des séquences ou des pans de conduite bien identifiés.

21 Mais il faut cependant bien voir que l'expression « éthique appliquée » est redondante : par définition, l'éthique a pour champ de recherche la conduite. Il ne fait pas sens, rigoureusement parlant, de vouloir qualifier d «'appliquée » l'éthique qui, par essence, doit l'être déjà. Par ailleurs, l'éthique ne peut pas, non plus, ne pas être connaissance et donc théorique. Un courant en éthique de la communication des organisations a fait preuve, durant quelque temps, d'un certain anti-intellectualisme en refusant toute référence aux théories et philosophies éthiques. La situation commence maintenant à évoluer dans un sens plus harmonieux: de nombreux chercheurs tentent de mieux utiliser l'éthique théorique sur des problèmes néanmoins extrêmement concrets des activités de communication des organisations.

Pratt et Rentner (1989) déplorent l'ignorance de la philosophie dans laquelle sont menées certaines recherches d'éthique en relations publiques et plaident pour une meilleure utilisation de cette ressource théorique :

There are no references to ethical theory, no attempts to offer a groundwork for ethical decision-making, and no integration of «public relations ethics» into the substantial literature of ethical inquiry.

It seems strange that while public relations has been sensitive to developing theoretical underpinning in other areas, it has ignored an already wealth of 
potentially useful theoretical discourse on ethics. (58) Bivins (1989) fait le même double constat :

The problem seems to be that... there is no accepted conceptual framework from which to study public relations ethics.

(...)

Given the need to strengten ethical reasoning abilities of future practitioners, even

a brief detour into the realm of moral philosophy would be helpful. (49-50)

Hunter (1990) fait valoir le même point mais plus largement au sujet de l'éthique des entreprises et en attribuant à la communication une fonction de relation entre la philosophie et les affaires :

Ethics encompasses lasting philosophical principles that do not change from one case to another. The philosopher has the philosophical background with no business expertise ; the businessman often has no philosophical background in ethics. Business ethics needs to be taught by the communication teacher who understands business and ethical principles. (5)

24 Une fois reconnue l'importance des fondements philosophiques dans la recherche en éthique de la communication des organisations, quelques tentatives d'utilisation effective de matériaux théoriques sont effectuées. Un grand nombre de chercheurs, par exemple Harcourt (1990) proposent ainsi d'appliquer des éléments de philosophies éthiques comme la règle (ou médiane) d'or d'Aristote, l'impératif catégorique de Kant, le principe de l'utilité sociale. D'autres, parmi lesquels Spencer et Lehman (1990) suggèrent le recours plus intégral à des approches ou théories éthiques comme l'utilitarisme, la théorie des devoirs (theory of duties), le situationnisme et la théorie de la justice comme équité de Rawls. Finalement, quelques-uns s'essaient à présenter une vue d'ensemble systématique des différentes théories éthiques ; ainsi, Lewis et Speck (1990) proposent un tableau où sont répertoriées, sous les deux catégories des approches téléologique et déontologique, plus d'une soixantaine de théories éthiques philosophiques.

L'éthique de la communication des organisations est une entreprise, difficile certes et qui reste à être menée à terme, mais néanmoins sensée dans la mesure où elle s'enracine dans un développement théorique, c'est-à-dire une réflexion rationnelle et à visée cognitive sur la conduite humaine. C'est seulement par un tel effort réflexif que pourrait être comprise et analysée la contradiction supposée entre l'intention morale et la finalité organisationnelle.

\section{L'analyse du raisonnement éthique}

Mais l'investigation philosophique ne peut d'elle-même suffire à cette tâche. Encore fautil qu'elle débouche sur une façon de faire spécifique : l'analyse du raisonnement éthique. Dire que l'éthique est une connaissance portant sur la conduite, c'est impliquer que celleci n'est pas (ou pas totalement) tributaire de l'arbitraire, que la conduite n'est pas qu'affaire de force, de pulsion ou d'émotion. C'est par le fait même également impliqué qu'une certaine forme de rationalité peut présider à l'action humaine, que les choix d'ordre moral sont effectués suivant un certain mode de raisonnement.

S'ouvre de la sorte la possibilité de poursuivre un type de travail tout à fait particulier : l'analyse du raisonnement éthique et, par extension, du jugement éthique. Ce travail a déjà été entrepris sur le plan général de la communication publique. Il fait aujourd'hui l'objet d'un certain intérêt pour le champ plus spécifique de l'éthique de la communication des organisations, avec l'ajout de deux nouvelles considérations propres à 
l'approche plus pragmatique par laquelle il se distingue: la prise de décision (decisionmaking) et la résolution de problèmes (problem-solving).

Il faut bien voir que l'analyse du raisonnement éthique, telle que l'appellent de leurs voeux les chercheurs qui l'évoquent, se situent dans le prolongement de la nécessité d'élaborer une théorie morale sur des bases philosophiques. Comme l'écrivent Pratt et Rentner (1989),...

If ethical decision-making is an inherently important component of a model of professionalism in general and public relations professionalism in particular, then public relations scholars, students, and practitioners should be familiar with the tools of the philosopher. "Reinventing the wheel » has always been a waste of valuable, limited resources. (59)

De la même façon, la raison pour laquelle Bivins (1989) fait valoir l'importance de la philosophie éthique est l'éclairage qu'elle peut apporter sur le processus de prise de décision éthique (ethical decison-making process).

De leur côté, quand ils en arrivent à proposer une synthèse de la théorie utilitariste et de la théorie des devoirs - proposition qui sera plus loin examinée avec plus de précision-, Spencer et Lehman (1990) le font dans la perspective très précise que cette unification serve de plate-forme à l'analyse du raisonnement et du jugement éthique (as a framework for decision-making and for supporting decisions...).

31 Il importe de préciser que l'analyse du raisonnement éthique relève d'un type bien particulier d'éthique: la métaéthique. Distinctement de l'éthique normative et de l'éthique descriptive, la métaéthique est une entreprise de deuxième niveau: elle se donne pour tâche d'étudier les fondements et les modes de fonctionnement de la théorie et du discours éthiques. L'analyse des procédés de raisonnement qui président à la prise de décision morale appartient manifestement à la métaéthique ${ }^{6}$.

Jusqu'à maintenant, deux modèles ou grilles d'interprétation ont été élaborés en analyse du raisonnement éthique : la Potter Box de Christians, Rotzoll et Fackler (1991) et la technique d'analyse éthique dans l'enseignement de la communication des affaires de Spencer et Lehman (1990).

D'abord mise au point pour l'éthique des pratiques traditionnelles de communication publique, le journalisme, la publicité, les médias et autres formes de divertissement (entertainment), la Potter Box fait depuis peu l'objet d'une application en relations publiques et en communication organisationnelle. Elle permet de reconstruire les raisonnements qui peuvent prendre prise sur les diverses alternatives morales. La Potter Box permet donc de mener de véritables analyses de cas en éthique de la communication des organisations. Plus spécifiquement, elle permet d'identifier les éléments moraux pertinents d'une situation, les valeurs et les principes éthiques en cause ainsi que les allégeances qu'entraînent les différents choix possibles.

De façon distincte mais dans un esprit apparenté, la technique d'analyse éthique dans l'enseignement de la communication des affaires de Spencer et Lehman permet de mettre au jour les différents aspects qui entrent en ligne de compte dans la résolution des dilemmes moraux en communication des entreprises ainsi que les différents éléments pouvant fonder une prise de position donnée. Elle comporte trois étapes successives : une description de cas, la détermination de son incidence éthique précise (en fonction d'une analyse utilitariste des « coûts et bénéfices » et d'une " évaluation d'impact » par rapport à des devoirs) et une justification de la décision effectivement retenue. 
grand intérêt de ces deux modèles est de permettre de tenir compte du caractère individuel et subjectif de l'éthique, eu égard plus spécifiquement au domaine des valeurs, sans pour autant dénier la possibilité d'une étude éthique objective. L'analyse du raisonnement éthique prend comme point de départ l'adhésion des individus à un certain système ou à une échelle de valeurs pour ensuite tenter d'en examiner les implications et conséquences. Si elles permettent ainsi de mesurer la rationalité des décisions à incidence morale prises par des agents, la Potter Box et la technique d'analyse éthique dans l'enseignement de la communication des affaires de Spencer et Lehman ne cherchent pas à fournir une évaluation proprement morale de ces choix. A fortiori, ces deux grilles d'analyse n'ont pas pour objectif d'amener les agents à changer leurs valeurs. Comme l'indiquent en toutes lettres Spencer et Lehman au sujet plus spécifiquement de l'enseignement de l'éthique de la communication des affaires, il ne s'agit jamais pour un professeur d'inculquer des valeurs à des étudiants mais plutôt de leur fournir des instruments pouvant leur permettre de mieux aborder les dilemmes moraux :

As a business communication professor, you cannot improve your students'personal morals. They corne to you with their own sensés of right and wrong already instilled. However, what you can do is to give them tools to help them deal with potentially unethical situations in the workplace. (7)

\section{Une éthique de la persuasion}

La communication ce fut d'abord l'information. La première pratique de communication historiquement reconnue fut le journalisme. Aussi n'est-il pas étonnant que la théorie de la communication, et en particulier les sous-théories des formes subséquentes de communication: la publicité, les relations publiques et aujourd'hui la communication organisationnelle et institutionnelle, furent fortement marquées par la théorie du journalisme. C'est aussi vrai pour le domaine éthique : l'éthique de ces autres formes de communication s'est jusqu'à maintenant construite, pour une large part, en référence à l'éthique journalistique.

Or, se cache peut-être là un vice de forme qui handicape la pleine reconnaissance et le plein développement de l'éthique de la communication des organisations. C'est du moins ce que soutient McBride (1989), nommément pour les seules relations publiques. Sa thèse peut cependant sans difficulté être étendue à l'ensemble des pratiques de communication autres que le journalisme, y compris la communication des organisations.

McBride prétend que l'éthique particulière du journalisme, qui s'articule autour de l'objectivité, est inappropriée et "dysfonctionnelle» pour l'éthique des relations publiques. Les deux pratiques de communication constituent, selon elle, deux mondes distincts dont l'associai ton a eu des conséquences néfastes pour l'éthique des relations publiques :

Practioners can only be outcasts in a journalistic culture.... there is a cultural chasm between journalism and public relations with implications for ethical practice neither fully acknowledged nor understood. (9)

Après avoir mis en évidence que l'attachement du journalisme à la norme d'objectivité a contribué, paradoxalement, à l'émergence et à l'extension des relations publiques, McBride suggère de dégager l'éthique des relations publiques de l'éthique du journalisme. La proposition précise qu'elle met ainsi de l'avant est de remplacer l'objectivité par la persuasion comme pierre angulaire de la moralité relationniste. Au lieu de continuer de 
chercher à se déterminer par rapport au journalisme, les relations publiques devraient, sans fausse honte, reconnaître leur objectif de défense d'un certain point de vue (advocacy) et en tirer les conséquences qui s'imposent. Dédouanées de l'objectivité journalistique et affichant clairement leur finalité persuasive, les relations publiques seraient à même, de l'avis de McBride, de mieux cerner leurs balises éthiques et aussi d'identifier leur responsabilité sociale spécifique. Encore une fois, il est tout à fait possible de prétendre la même chose pour la communication organisationnelle dans son ensemble.

40 Encore plus que les points précédemment développés, cette idée de faire reposer l'éthique de la communication des organisations sur son objectif de persuasion, pourrait faciliter la résolution de l'apparente contradiction entre éthique et organisations. En fait, cette antinomie reste tributaire d'une considération limitée de la moralité de laquelle serait exclue la persuasion conçue comme étant au mieux amorale, au pire immorale. Dans la perspective inverse, une fois bien admise la nature intéressée de l'activité des organisations et donc leur souci d'efficacité et même de rentabilité, et ouverte la possibilité de poser la question éthique à leur finalité persuasive, le paradoxe tombe de lui-même.

41 L'éthique de persuasion que McBride appelle de ses voeux est en fait déjà en cours d'élaboration. Des chercheurs appartenant à ce qu'on peut appeler «l'école rhétorique américaine contemporaine», comme Sproule (1980) et Jensen (1981), explorent la question de la moralité de l'argumentation communicationnelle. Leur préoccupation essentielle porte sur la nature et la forme des différents types d'arguments qui sont utilisés dans les pratiques de communication publique et plus largement dans toutes les formes d'expression à visée interpersonnelle. Comme par ailleurs ils reconnaissent explicitement la finalité persuasive de la communication, ils sont amenés à traiter de sa dimension éthique. Pour eux, la question prend la formulation plus précise suivante: quels sont les critères en vertu desquels les arguments, ou plus spécifiquement les utilisations possibles d'arguments peuvent être reconnues comme étant morales.

\section{L'importance de l'intérêt (self-interest)}

Dans le prolongement de l'intuition de développer, pour l'éthique de la communication des organisations, une éthique de la persuasion, est souvent faite l'affirmation d'y tenir compte de la notion d'intérêt. Dans quelques travaux consacrés à l'éthique de la communication des organisations, il est déjà fait une bonne place aux intérêts des agents et des organisations elles-mêmes.

Lewis et Speck (1990) font ressortir de leur considération historique des doctrines éthiques le principe de l'hédonisme psychologique (psychological hedonism) suivant lequel une prescription morale acquiert sa validité du fait que ceux qui y adhèrent $\mathrm{y}$ trouvent leur compte. Dans cette perspective, c'est l'auto-réalisation (self-realization) qui fournit aux agents l'ultime raison d'adopter un comportement moral :

... the ethical (i.e. the right, good, moral, just) life "is defined" in terms of what is life-enhancing or beneficial to life, both for oneself and for others, both now and in the future. Conversely, the unethical (i.e., the wrong, bad, immoral, unjust) life is defined in terms of what is life-threatening or detrimental to life, both to oneself and to others, both in the present and in the future. 
While this definition does not explain in every case precisely what is ethical or unethical behavior, it does explain why the individual should pursue the ethical life. It is in his or her best self-interest. (222) théorique, dans une structure hiérarchique différente de l'ordre séquenciel suivant lequel elles ont été ici exposées. C'est essentiellement l'analyse métaéthique du raisonnement éthique qui constitue la voie de reconnaissance de l'éthique de la communication des organisations. La thèse de l'inhérence et l'appui sur l'éthique théorique et philosophique sont en fait des préalables qui permettent de mieux fonder l'analyse du raisonnement éthique. Quant à la démarcation de l'éthique de la persuasion de la communication des organisations de l'éthique de l'objectivité du journalisme et la prise en compte de l'intérêt des agents, elles viennent spécifier dans quelle direction plus précise pourrait être poursuivi le projet d'une éthique normative de la communication des organisations.

Seule la poursuite effective de ce projet pourra en démontrer la réalité ou la nature chimérique. Sa dénonciation à priori est prématurée. 


\section{BIBLIOGRAPHIE}

Bivins, Thomas H. (1989) : Are Public Relations Texts Covering Ethics Adequately?, Journal of Mass Media Ethics, vol. 4, n 1, 39-52.

Christians, Clifford G. ; Rotzoll, Kim B. ; Fackler, Mark (1991) : Media Ethics. Cases \& Moral Reasoning, Philadelphia : Longman, third edition (1987 et 1989).

Gauthier, Gilles (1991a) : Communication publique, éthique et société. Communication publique et Société. Repères pour la réflexion et l'action, Montréal : Gaëtan Morin éditeur.

(1991b) : L'exigence éthique de la communication publique, Ethica, vol. 2, nº l,

(1991c) : Éthique de la communication et entreprise. Pour une métaéthique : l'analyse du raisonnement et du jugement éthique (La recherche nord-américaine contemporaine) , éthique \& Communication Actes du 5ème Colloque Entreprise et Communication, Textes réunis par Hugues Hotier, Bordeaux : Groupe de Recherche en Communication des Organisations Entreprise et Communication GREC/O et Institut des Sciences de l'Information et de la Communication Université Michel de Montaigne Bordeaux 3,199-221.

Goodrich, H ; (1958) : Morality in the teaching of letter writing, ABWA Bulletin, vol. 22, n 8, 9-11.

Harcourt, Jules (1990) : Developing Ethical Messages : A Unit of Instruction for the Basic Business Communication Course, The Bulletin of the Association for Business Communication, vol. 53, $\mathrm{n}$ -3,17-20.

Hotier, Hugues (1991) : Vous avez dit "éthique»?, éthique \& Communication Actes du 5 e Colloque Entreprise et

Communication, Textes réunis par Hugues Hotier, Bordeaux : Groupe de Recherche en Communication des Organisations Entreprise et Communication GREC/O et Institut des Sciences de l'Information et de la Communication Université Michel de Montaigne Bordeaux 3,17-25.

Hunter, Jennie (1990) : Business Ethics : Who Cares?, The Bulletin of the Association for Business Communication, vol. 53, n 3,4-6.

Jacobi (1990) : Using the Enthymeme to Emphasize Ethics in Professional Writing Courses, The Journal of Business Communication, vol. 27, n 3,273-292.

Jensen, J. (1981) : Argumentation. Reasoning in Communication, New York : Van Nostrand.

Johannesen, Richard (1990) : Ethics in Human Communication, Prospect Heights, Illinois, Waveland Press Inc., third edition (1983 et 1975).

Jones, Donald G. (1977) : A Bibliography of Business Ethics, 1975-1981, Charlottesville : University Press of Virginia.

Jones, Donald G. ; Bennet, Patricia (1986) : A Bibliography of Business Ethics, 1981-1985, Levinston : The Edwin Mellen Press.

Lewis, Phillip V. ; Speck, Henry E. III (1990) : Ethical Orientations for Understanding Business Ethics, The Journal of Business Communication, vol. 27, $\mathrm{n}^{\circ} 3,213-232$.

McBride, Genevieve (1989) : Ethical Thought in Public Relations History : Seeking a Relevant Perspective, Journal of Mass Media Ethics, vol. 4, n 1, 5-20. 
McGuire, R.L. (1980) : Can we teach ethics. We do teach ethics, Unfurling communication's colors in the 80's. Proceedings of the American Business Communication Association, Bruno, S.J. (ed.), Texas : School of Business and Public Administration, University of Houston-Clear Lake, 113-20.

Murphy, Patrick E. ; Bloom, Paul N. (1990) : Ethical Issues in Social Marketing, Fine, Seymour H. (ed.) : Social Marketing. Promoting the Causes of Public and Nonprofit Agencies, Boston : Allyn \& Bacon, 68-78.

Pettit, John N. J.-R. ; Vaught, Bobby ; Pulley, Kathy J. (1990) : The Role of Communication in Organisations : Ethical Considerations, The Journal of Business Communication, vol. 27, $\mathrm{n}^{\circ}$ 3,233-49.

Pratt, Catherine A. ; Rentner, Terry Lynn (1989) : What s Really Being Taught About Ethical Behavior, Public Relations Review, vol. 15, n 1, 53-66.

Reinsch, N. L. Jr. (1990) : Ethics Research in Business Communication : The State of the Art, The Journal of Business Communication, vol. 27, $\mathrm{n}^{\circ}$ 3, 251-272.

Rents, K.C. ; Debs, M.B. (1987) : Language and Corporate values: Teaching ethics in business writing courses, The Journal of Business Communication, vol. 8, $\mathrm{n}^{\circ}$ 4, 37-51.

Spencer, Barbara A. ; Lehman, Carol M. : Analysing Ethical Issues : Essential Ingredient in the Business Communication Course of the 1990s, The Bulletin of the Association for Business Communication, vol. 53, no $3,7-16$.

Sproule, Michael J. (1980) : Argument Language and Its Influence, New York : McGraw-Hill.

\section{NOTES}

1. C'est, me semble-t-il, l'attitude "intellectuelle» la plus répandue aujourd'hui en France. Je n'en donnerai, à titre symptomatique, qu'un seul exemple. Lors du 5ème colloque Entreprise et Communication organisé psr l'ISIC en novembre 1990 sur le thème éthique et Communication, Hugues Hotier posant la problématique mettait en avant deux hypothèses :

Hypothèse 1 Dans les organisations, l'éthique n'existe que comme outil de management ou, pour reprendre l'excellente expression de Jean Padio-Ieau, « une clef stratégique pour la survie et la rentabilité des firmes engagées dans une concurrence farouche et mondiale ». (...) Hypothèse 2 L'éthique n'est pas un principe unique, transcendant. Il y a autant d'éthiques que de formes de la communication et pour ce qui est des organisations, presque autant d'éthiques que d'entreprises. C'est à dire que l'éthique est un avatar et un vecteur de l'idéologie de l'entreprise, queue que soit cette idéologie. Hotier(1991), 24. Le moins que l'on puisse dire c'est que ces « hypothèse » ne sont pas innocentes. Elles impliquent a priori le rejet de toute caractérisa bon positive de l'éthique de la communication des organisations.

2. Hugues Hotier rapporte un exemple de définition manifestement inappropriée parce que trop large : « Tensemble des règles d'échange dans l'entreprise qui permettent à un groupe d'exister durablement » Hotier(1991),19.Au cours du colloque mentionné ci-avant, différents élément » de définition de l'éthique ont été lance», y compria des plus saugrenus, l'un par exempte relativement au « charisme du chef d'entreprise »!

3. Voir, à et sujet, entre autres choies Gauthier(1991a).

4. Voir Jones (1977) et Jones et Bennet (1986).

5. J'ai tenté ailleurs « de caractériser les différente formulations possibles " de la thèse de l'inhérence. Voir Gauthier (1991b)

6. Pour une discus-sion plus dlétaillée des différences entre éthique normative, éthique descriptive et métaéthique, voir Gauthier (1991c). 


\section{RÉSUMÉS}

Le texte se veut une "défense et illustration» de l'importance, théorique et pratique, de l'éthique de la communication des organisations. Il prétend dissiper une prétendue opposition entre l'intention morale et la visée organisationnelle. Cinq conditions sont analysées qui permettent de Justifier l'éthique de la communication des organisations: la thèse dite «de l'inhérence ", la nécessité de fondements, théorico-philosophiques, l'analyse du raisonnement éthique, l'admission d'une finalité persuasive et la reconnaissance de l'intérêt.

\section{AUTEUR}

\section{GILLES GAUTHIER}

Gilles Gauthier est professeur au département d'information et de communication de l'Université Laval à Québec. Il a fait paraître une douzaine de textes en éthique de la communication publique. Ses autres champs de recherche portent sur la communication politique, la philosophie et la pratique du journalisme et l'argumentation dans la communication publique. 